\title{
RATIONAL WAVE SOLUTIONS TO A GENERALIZED (2+1)-DIMENSIONAL HIROTA BILINEAR EQUATION
}

\author{
K. Hosseini ${ }^{1}$, M. Mirzazadeh ${ }^{2, *}$, M. Aligoli ${ }^{3}$, M. Eslami $^{4}$ \\ AND J.G. LIU ${ }^{5}$
}

\begin{abstract}
A generalized form of (2+1)-dimensional Hirota bilinear (2D-HB) equation is considered herein in order to study nonlinear waves in fluids and oceans. The present goal is carried out through adopting the simplified Hirota's method as well as ansatz approaches to retrieve a bunch of rational wave structures from multiple soliton solutions to breather, rational, and complexiton solutions. Some figures corresponding to a series of rational wave structures are provided, illustrating the dynamics of the obtained solutions. The results of the present paper help to reveal the existence of rational wave structures of different types for the 2D-HB equation.
\end{abstract}

Mathematics Subject Classification. 45K05; 83C15.

Received January 27, 2020. Accepted May 2, 2020.

\section{INTRODUCTION}

One of the attractive subjects in the areas of mathematical physics is to look for rational wave solutions of differential equations. There are different rational wave solutions to differential equations; for example, multiple soliton solutions, breather solutions, rogue solutions, and so on. During the last decades, many effective methods such as inverse scattering method [4], Hirota's direct method [11], multiple exp-function method [1, 15, 21, 22], simplified Hirota's method [9, 10, 12, 27, 28, 30-33], and ansatz methods [2, 13, 25, 26, 29] have been exerted to find rational wave solutions of differential equations. More articles can be found in [3, 5-8, 16-19, 23, 24, 34, 35].

The basic goal of this paper is to study a generalized 2D-HB equation describing nonlinear waves in fluids and oceans as follows [14]

$$
\phi_{y t}+c_{1}\left(\phi_{x x x y}+6 \phi_{x} \phi_{y}+3 \phi_{x y} \phi+3 \phi_{x x} \int \phi_{y} \mathrm{~d} x\right)+c_{2} \phi_{y y}=0
$$

Keywords and phrases: (2+1)-dimensional Hirota bilinear equation, generalized form, simplified Hirota's method, ansatz approaches, multiple soliton, breather, rational, and complexiton solutions.

1 Department of Mathematics, Rasht Branch, Islamic Azad University, Rasht, Iran.

2 Department of Engineering Sciences, Faculty of Technology and Engineering, East of Guilan, University of Guilan, P.C. 44891-63157 Rudsar-Vajargah, Iran.

3 Department of Applied Mathematics, Faculty of Mathematical Sciences, University of Guilan, PO Box 41335-1914 Guilan, Rasht, Iran.

4 Department of Mathematics, Faculty of Mathematical Sciences, University of Mazandaran, Babolsar, Iran.

5 College of Computer, Jiangxi University of Traditional Chinese Medicine, Jiangxi 330004, PR China.

* Corresponding author: mirzazadehs2@gmail.com 
or

$$
\phi_{y t}+c_{1}\left(\phi_{x x x y}+6 \phi_{x} \phi_{y}+3 \phi_{x y} \phi+3 \phi_{x x} \psi\right)+c_{2} \phi_{y y}=0, \quad \phi_{y}=\psi_{x}
$$

which is a generalization of the Hirota bilinear equation studied in [20]. The Hirota bilinear form corresponding to the above nonlinear model (1.1) is

$$
\begin{aligned}
B_{H B E}(\varphi):= & \left(D_{y} D_{t}+c_{1} D_{x}^{3} D_{y}+c_{2} D_{y}^{2}\right) \varphi \cdot \varphi=2\left(\varphi \varphi_{y t}-\varphi_{y} \varphi_{t}+c_{1}\left(\varphi \varphi_{x x x y}-3\left(\varphi_{x x y} \varphi_{x}-\varphi_{x y} \varphi_{x x}\right)-\varphi_{y} \varphi_{x x x}\right)\right. \\
& \left.+c_{2}\left(\varphi \varphi_{y y}-\varphi_{y}^{2}\right)\right)=0,
\end{aligned}
$$

under the specific transformation

$$
\phi=2(\ln \varphi)_{x x}
$$

Recently, Hua et al. investigated the interaction behavior associated with the above generalized 2D-HB equation in [14]. They constructed two types of interaction solutions through adopting different test functions as below

$$
\varphi=g^{2}+h^{2}+k e^{l}+a_{12}, \quad \text { and } \quad \varphi=g^{2}+h^{2}+\cosh (l)+a_{12},
$$

where

$$
g=a_{1} x+a_{2} y+a_{3} t+a_{4}, \quad h=a_{5} x+a_{6} y+a_{7} t+a_{8}, \quad l=a_{9} x+a_{10} y+a_{11} t
$$

In order to advance the studies on the generalized 2D-HB equation (1.1); in this paper, the simplified Hirota's method as well as different ansatz approaches are utilized formally to retrieve a bunch of rational wave structures from multiple soliton solutions to breather, rational, and complexiton solutions.

\section{The Generalized 2D-HB EQUATION AND ITS RATIONAL WAVES SOLUTIONS}

In this section, a number of rational wave structures from multiple soliton solutions to breather, rational, and complexiton solutions for the generalized 2D-HB equation are derived using the simplified Hirota's method as well as ansatz approaches.

\subsection{Multiple soliton solutions}

In order to procure multiple soliton solutions of the generalized 2D-HB equation, first the expression

$$
\phi=e^{\theta_{i}}, \quad \theta_{i}=\kappa_{i} x+\tau_{i} y-\varsigma_{i} t
$$

is substituted into the linear terms of the generalized 2D-HB equation; and then, the resulting equation is solved for obtaining the dispersion relation $\varsigma_{i}$. This leads to

$$
\varsigma_{i}=c_{1} \kappa_{i}^{3}+c_{2} \tau_{i}
$$

and therefore, the phase variables $\theta_{i}, \quad 1 \leq i \leq N$ can be written as

$$
\theta_{i}=\kappa_{i} x+\tau_{i} y-\left(c_{1} \kappa_{i}^{3}+c_{2} \tau_{i}\right) t, \quad 1 \leq i \leq N
$$


Now, by inserting the logarithmic transformations

$$
\phi=R(\ln \varphi)_{x x}, \quad \psi=R(\ln \varphi)_{x y}
$$

into (1.2) where the auxiliary function $\varphi(x, y, t)$ is defined as

$$
\varphi=1+e^{\kappa_{1} x+\tau_{1} y-\left(c_{1} \kappa_{1}^{3}+c_{2} \tau_{1}\right) t},
$$

we find that $R=2$.

Based on this result, a single soliton solution can be obtained as

$$
\begin{gathered}
\phi(x, y, t)=\frac{2 \kappa_{1}^{2} e^{\kappa_{1} x+\tau_{1} y-\left(c_{1} \kappa_{1}^{3}+c_{2} \tau_{1}\right) t}}{1+e^{\kappa_{1} x+\tau_{1} y-\left(c_{1} \kappa_{1}^{3}+c_{2} \tau_{1}\right) t}}-\frac{2 \kappa_{1}^{2} e^{2\left(\kappa_{1} x+\tau_{1} y-\left(c_{1} \kappa_{1}^{3}+c_{2} \tau_{1}\right) t\right)}}{\left(1+e^{\kappa_{1} x+\tau_{1} y-\left(c_{1} \kappa_{1}^{3}+c_{2} \tau_{1}\right) t}\right)^{2}} \\
\psi(x, y, t)=\frac{2 \kappa_{1} \tau_{1} e^{\kappa_{1} x+\tau_{1} y-\left(c_{1} \kappa_{1}^{3}+c_{2} \tau_{1}\right) t}}{1+e^{\kappa_{1} x+\tau_{1} y-\left(c_{1} \kappa_{1}^{3}+c_{2} \tau_{1}\right) t}}-\frac{2 \kappa_{1} \tau_{1} e^{2\left(\kappa_{1} x+\tau_{1} y-\left(c_{1} \kappa_{1}^{3}+c_{2} \tau_{1}\right) t\right)}}{\left(1+e^{\kappa_{1} x+\tau_{1} y-\left(c_{1} \kappa_{1}^{3}+c_{2} \tau_{1}\right) t}\right)^{2}} .
\end{gathered}
$$

For the double soliton solution, the following auxiliary function is considered

$$
\varphi=1+e^{\theta_{1}}+e^{\theta_{2}}+a_{12} e^{\theta_{1}+\theta_{2}},
$$

in which the phase variables $\theta_{i}, i=1,2$ are as before and the phase shift $a_{12}$ is an unknown. After performing some calculations, a double soliton solution is gained as

$$
\phi=2(\ln \varphi)_{x x}, \quad \psi=2(\ln \varphi)_{x y}
$$

where the phase shift $a_{12}$ is

$$
a_{12}=\frac{\kappa_{1} \tau_{1}-\kappa_{1} \tau_{2}-\kappa_{2} \tau_{1}+\kappa_{2} \tau_{2}}{\kappa_{1} \tau_{1}+\kappa_{1} \tau_{2}+\kappa_{2} \tau_{1}+\kappa_{2} \tau_{2}} .
$$

Finally, a triple soliton solution to the generalized 2D-HB equation is derived as

$$
\phi=2(\ln \varphi)_{x x}, \quad \psi=2(\ln \varphi)_{x y},
$$

in which the auxiliary function, the phase shifts, and the phase variables are defined as

$$
\begin{gathered}
\varphi=1+e^{\theta_{1}}+e^{\theta_{2}}+e^{\theta_{3}}+a_{12} e^{\theta_{1}+\theta_{2}}+a_{13} e^{\theta_{1}+\theta_{3}}+a_{23} e^{\theta_{2}+\theta_{3}}+a_{12} a_{13} a_{23} e^{\theta_{1}+\theta_{2}+\theta_{3}}, \\
a_{i j}=\frac{\kappa_{i} \tau_{i}-\kappa_{i} \tau_{j}-\kappa_{j} \tau_{i}+\kappa_{j} \tau_{j}}{\kappa_{i} \tau_{i}+\kappa_{i} \tau_{j}+\kappa_{j} \tau_{i}+\kappa_{j} \tau_{j}}, \quad 1 \leq i, j \leq 3, \\
\theta_{i}=\kappa_{i} x+\tau_{i} y-\left(c_{1} \kappa_{i}^{3}+c_{2} \tau_{i}\right) t, \quad 1 \leq i \leq 3 .
\end{gathered}
$$

It is easy to show that the following multiple complex soliton solutions to the generalized 2D-HB equation can be constructed

$$
\phi_{1,2,3}=2\left(\ln \varphi_{1,2,3}\right)_{x x}, \quad \psi_{1,2,3}=2\left(\ln \varphi_{1,2,3}\right)_{x y}
$$



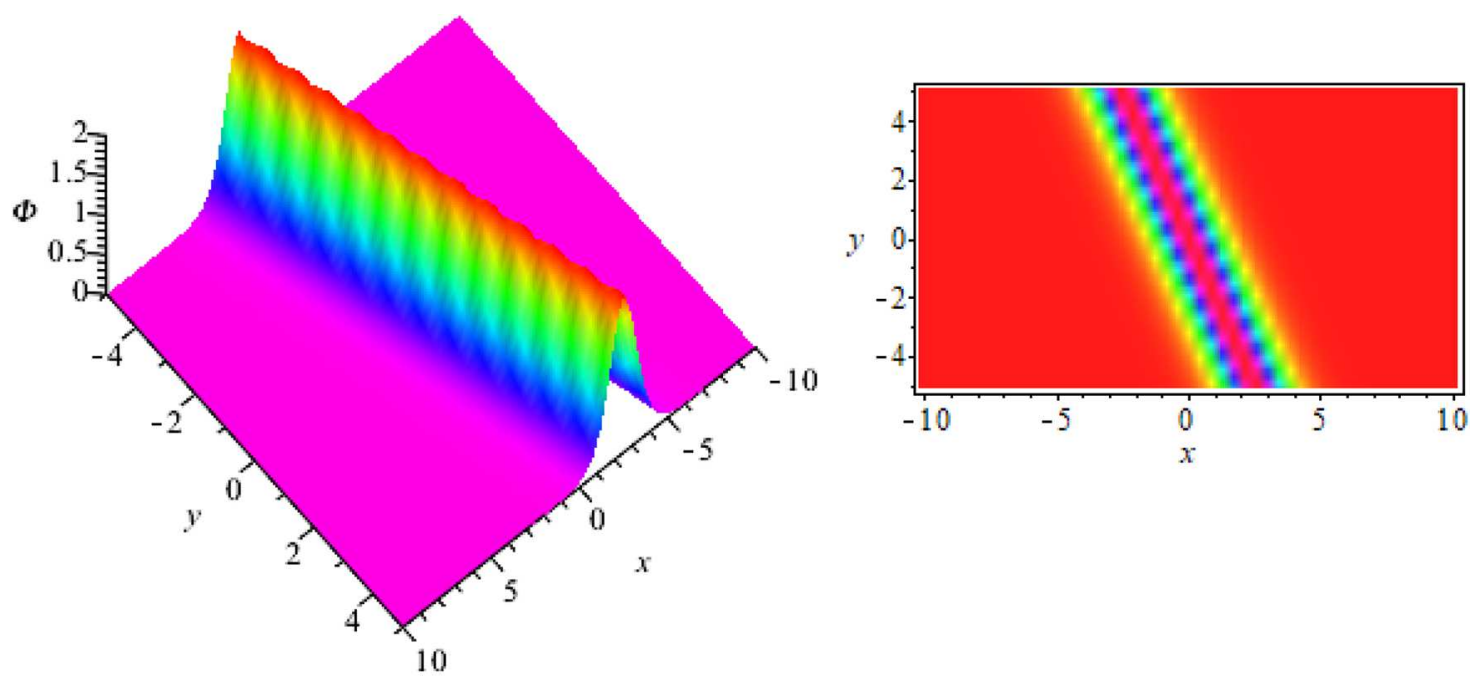

Figure 1. Single soliton solution when $\kappa_{1}=1, \tau_{1}=2$, and $t=0$.

in which

$$
\begin{gathered}
\varphi_{1}=I+e^{\theta_{1}}, \quad I=\sqrt{-1}, \\
\varphi_{2}=I+e^{\theta_{1}}+e^{\theta_{2}}-I a_{12} e^{\theta_{1}+\theta_{2}}, \quad I=\sqrt{-1}, \\
\varphi_{3}=I+e^{\theta_{1}}+e^{\theta_{2}}+e^{\theta_{3}}-I a_{12} e^{\theta_{1}+\theta_{2}}-I a_{13} e^{\theta_{1}+\theta_{3}}-I a_{23} e^{\theta_{2}+\theta_{3}}-a_{12} a_{13} a_{23} e^{\theta_{1}+\theta_{2}+\theta_{3}}, \quad I=\sqrt{-1},
\end{gathered}
$$

and

$$
\begin{array}{cl}
a_{i j} & =\frac{\kappa_{i} \tau_{i}-\kappa_{i} \tau_{j}-\kappa_{j} \tau_{i}+\kappa_{j} \tau_{j}}{\kappa_{i} \tau_{i}+\kappa_{i} \tau_{j}+\kappa_{j} \tau_{i}+\kappa_{j} \tau_{j}}, \quad 1 \leq i, j \leq 3, \\
\theta_{i} & =\kappa_{i} x+\tau_{i} y-\left(c_{1} \kappa_{i}^{3}+c_{2} \tau_{i}\right) t, \quad 1 \leq i \leq 3 .
\end{array}
$$

The plots of single, double, and triple soliton solutions have been provided in Figures $1-3$, illustrating the dynamics of the multiple solutions.

Figure 1 shows a bright soliton wave whereas Figure 2 demonstrates the interaction of two bright soliton waves. Furthermore, the interaction of three bright soliton waves including two strong waves and one weak wave has been illustrated in Figure 3.

\subsection{Breather and rational solutions}

In the present subsection, first, the breather solution of the generalized 2D-HB equation is acquired by adopting a specific ansatz approach which is a combination of exponential functions and a trigonometric function as follows

$$
\varphi=e^{-k X}+b_{0} \cos (h Y)+b_{1} e^{k X}
$$



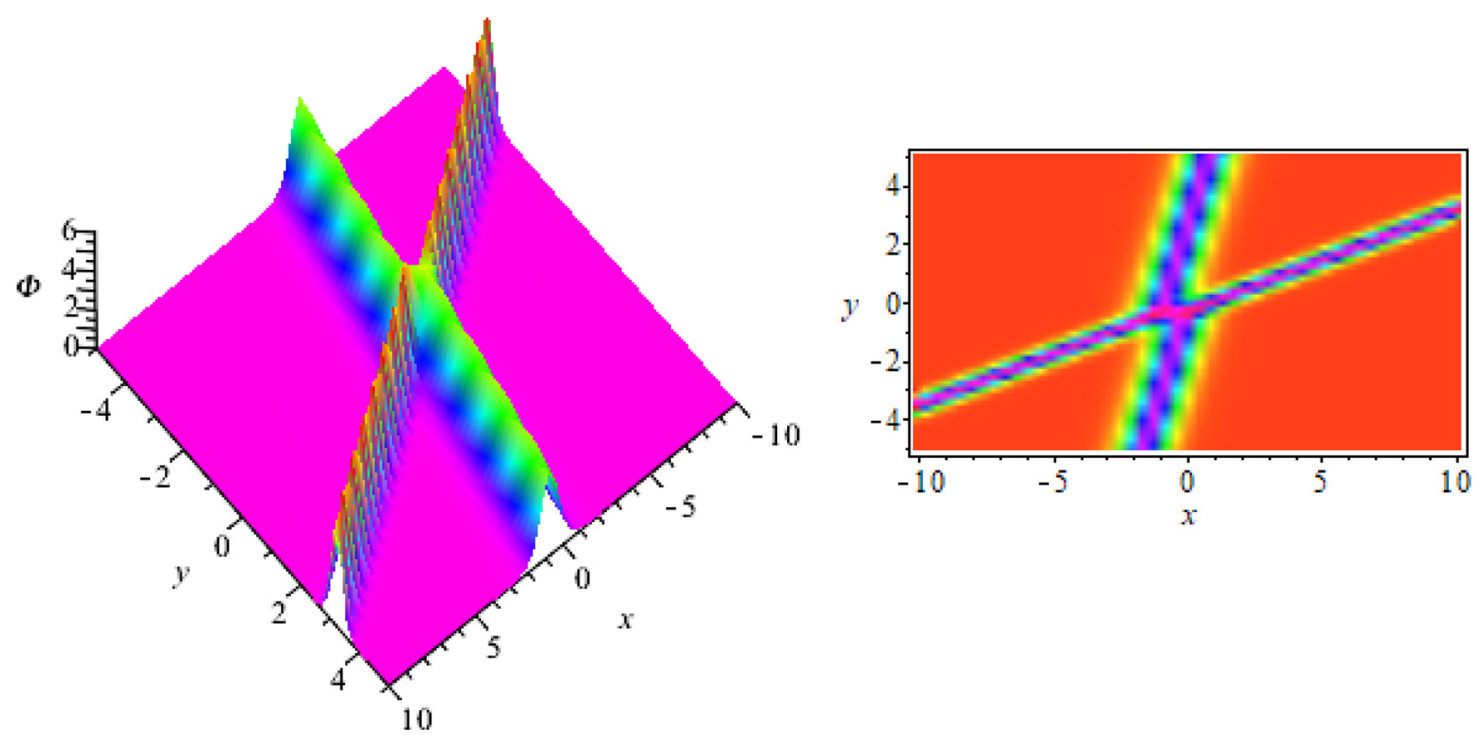

Figure 2. Double soliton solution when $\kappa_{1}=-3.5, \tau_{1}=10, \kappa_{2}=3, \tau_{2}=-1$, and $t=0$.
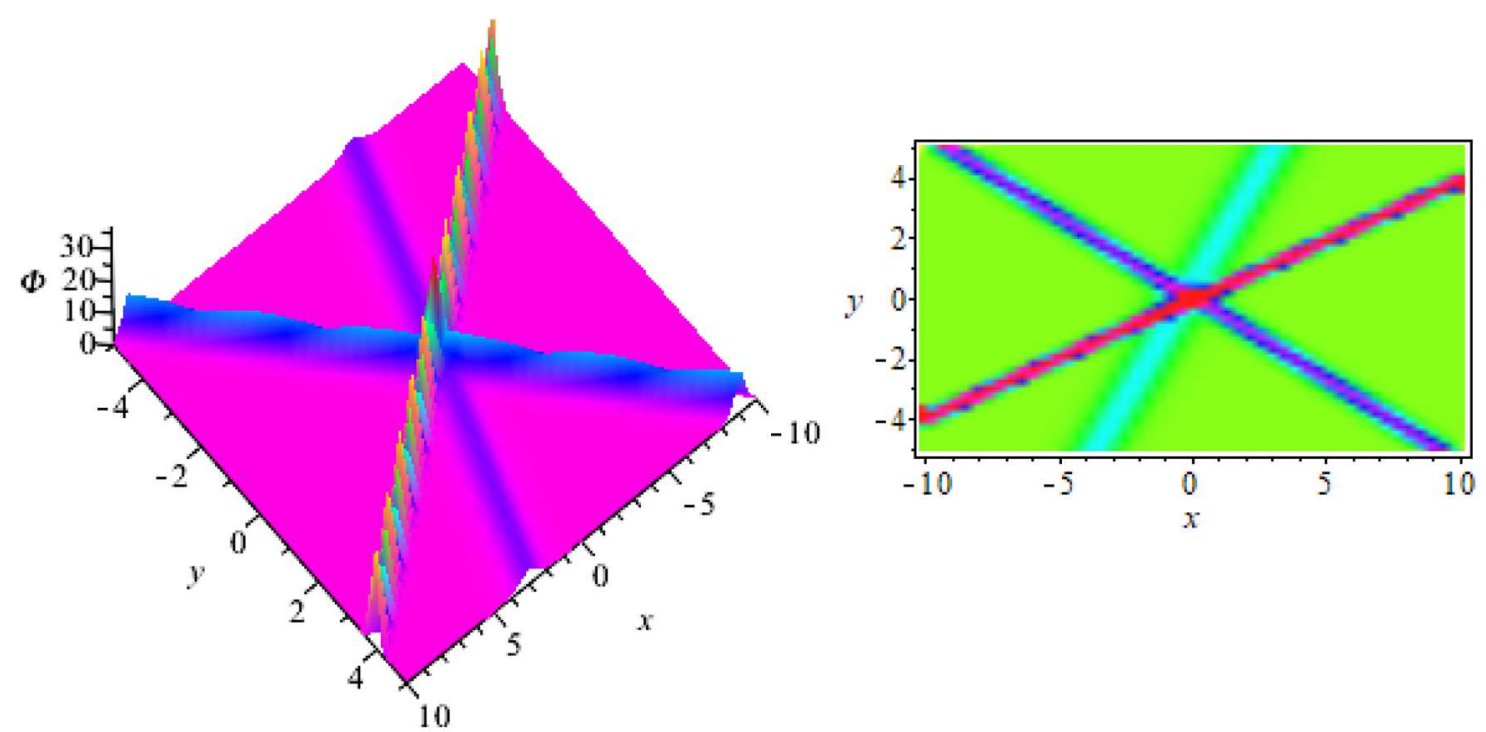

Figure 3. Triple soliton solution when $\kappa_{1}=3, \tau_{1}=-2, \kappa_{2}=-5, \tau_{2}=-9, \kappa_{3}=-8, \tau_{3}=20$, and $t=0$.

in which

$$
X=a_{1} x+a_{2} y+a_{3} t+a_{4}, \quad Y=a_{5} x+a_{6} y+a_{7} t+a_{8}
$$

and $k, b_{0}, h, b_{1}$ and $a_{i}, 1 \leq i \leq 8$ are constants to be computed. Inserting equation (2.21) into equation (1.3) and performing some calculations, yields the following nonlinear algebraic system

$$
\begin{aligned}
& 2 h^{4} a_{5}^{3} a_{6} b_{0} c_{1}-6 h^{2} k^{2} a_{1}^{2} a_{5} a_{6} b_{0} c_{1}-6 h^{2} k^{2} a_{1} a_{2} a_{5}^{2} b_{0} c_{1}+2 k^{4} a_{1}^{3} a_{2} b_{0} c_{1}-2 h^{2} a_{6}^{2} b_{0} c_{2}+2 k^{2} a_{2}^{2} b_{0} c_{2}-2 h^{2} a_{6} a_{7} b_{0} \\
& +2 k^{2} a_{2} a_{3} b_{0}=0
\end{aligned}
$$




$$
\begin{aligned}
& \quad 2 h^{4} a_{5}^{3} a_{6} b_{0} b_{1} c_{1}-6 h^{2} k^{2} a_{1}^{2} a_{5} a_{6} b_{0} b_{1} c_{1}-6 h^{2} k^{2} a_{1} a_{2} a_{5}^{2} b_{0} b_{1} c_{1}+2 k^{4} a_{1}^{3} a_{2} b_{0} b_{1} c_{1}-2 h^{2} a_{6}^{2} b_{0} b_{1} c_{2}+2 k^{2} a_{2}^{2} b_{0} b_{1} c_{2} \\
& -2 h^{2} a_{6} a_{7} b_{0} b_{1}+2 k^{2} a_{2} a_{3} b_{0} b_{1}=0 \\
& -6 h^{3} k a_{1} a_{5}^{2} a_{6} b_{0} b_{1} c_{1}-2 h^{3} k a_{2} a_{5}^{3} b_{0} b_{1} c_{1}+2 h k^{3} a_{1}^{3} a_{6} b_{0} b_{1} c_{1}+6 h k^{3} a_{1}^{2} a_{2} a_{5} b_{0} b_{1} c_{1}+4 h k a_{2} a_{6} b_{0} b_{1} c_{2}+2 h k a_{2} a_{7} b_{0} b_{1} \\
& +2 h k a_{3} a_{6} b_{0} b_{1}=0 \\
& 6 h^{3} k a_{1} a_{5}^{2} a_{6} b_{0} c_{1}+2 h^{3} k a_{2} a_{5}^{3} b_{0} c_{1}-2 h k^{3} a_{1}^{3} a_{6} b_{0} c_{1}-6 h k^{3} a_{1}^{2} a_{2} a_{5} b_{0} c_{1}-4 h k a_{2} a_{6} b_{0} c_{2}-2 h k a_{2} a_{7} b_{0}-2 h k a_{3} a_{6} b_{0}=0 \\
& \quad 8 h^{4} a_{5}^{3} a_{6} b_{0}^{2} c_{1}+32 k^{4} a_{1}^{3} a_{2} b_{1} c_{1}-2 h^{2} a_{6}^{2} b_{0}^{2} c_{2}-2 h^{2} a_{6} a_{7} b_{0}^{2}+8 k^{2} a_{2}^{2} b_{1} c_{2}+8 k^{2} a_{2} a_{3} b_{1}=0
\end{aligned}
$$

whose solution is

$$
a_{3}=3 h^{2} a_{1} a_{5}^{2} c_{1}-k^{2} a_{1}^{3} c_{1}-a_{2} c_{2}, \quad a_{7}=h^{2} a_{5}^{3} c_{1}-3 k^{2} a_{1}^{2} a_{5} c_{1}-a_{6} c_{2}, \quad b_{1}=-\frac{a_{5} h^{2} a_{6} b_{0}^{2}}{4 a_{1} k^{2} a_{2}} .
$$

Now, a breather solution is obtained as

$$
\phi=2(\ln \varphi)_{x x}, \quad \psi=2(\ln \varphi)_{x y}
$$

where

$$
\varphi=e^{-k\left(a_{1} x+a_{2} y+a_{3} t+a_{4}\right)}+b_{0} \cos \left(h\left(a_{5} x+a_{6} y+a_{7} t+a_{8}\right)\right)+b_{1} e^{k\left(a_{1} x+a_{2} y+a_{3} t+a_{4}\right)},
$$

and $a_{3}, a_{7}$, and $b_{1}$ have been defined in (2.28).

The plots of the breather solution (2.29) have been depicted in Figure 4, demonstrating its dynamical behavior. It is evident that Figure 4 presents the interaction of bright and dark waves with a periodic property.

Now, the rational solution is obtained from the breather solution (2.29) in a specific way.

Remark 2.1. It should be noted that by setting $a_{1}=a_{5}, a_{2}=-a_{6}, b_{0}=-2$, and $h=k$ in $(2.29)$ and letting $k \rightarrow 0$, a rational solution to the generalized 2D-HB equation can be constructed as

$$
\begin{gathered}
\phi(x, y, t)=8 a_{5}^{2}\left(\frac{1}{\mathcal{X}^{2}+\mathcal{Y}^{2}}-\frac{(\mathcal{X}+\mathcal{Y})^{2}}{\left(\mathcal{X}^{2}+\mathcal{Y}^{2}\right)^{2}}\right), \\
\psi(x, y, t)=\frac{8 a_{5} a_{6}\left(\mathcal{X}^{2}-\mathcal{Y}^{2}\right)}{\left(\mathcal{X}^{2}+\mathcal{Y}^{2}\right)^{2}}
\end{gathered}
$$

in which $\mathcal{X}=a_{5} x-a_{6} y+c_{2} a_{6} t+a_{4}$ and $\mathcal{Y}=a_{5} x+a_{6} y-c_{2} a_{6} t+a_{8}$.

To show it, by considering $a_{1}=a_{5}, a_{2}=-a_{6}, b_{0}=-2$, and $h=k$; it is obvious that the breather solution (2.29) can be written as

$$
\begin{gathered}
\phi(x, y, t)=\frac{2 a_{5}^{2} k^{2}(\cosh (k X)+\cos (k Y))}{\cosh (k X)-\cos (k Y)}-\frac{2\left(a_{5} k(\sinh (k X)+\sin (k Y))\right)^{2}}{(\cosh (k X)-\cos (k Y))^{2}} \\
\psi(x, y, t)=-2 a_{5} a_{6} k^{2}+\frac{2 a_{5} a_{6} k^{2}\left(\sinh ^{2}(k X)-\sin ^{2}(k Y)\right)}{(\cosh (k X)-\cos (k Y))^{2}}
\end{gathered}
$$



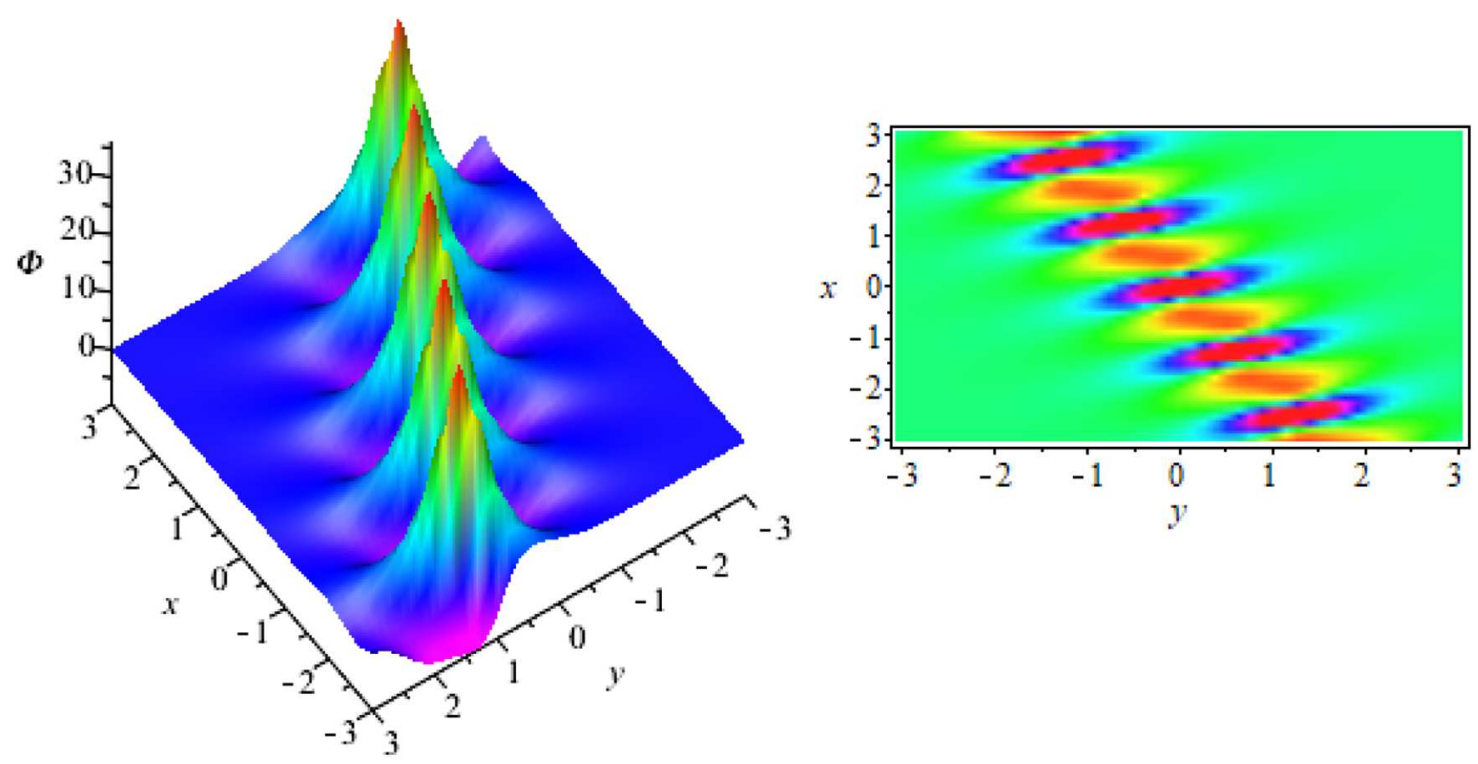

FiguRE 4. Breather solution when $a_{1}=1, a_{2}=2, a_{4}=0, a_{5}=-2, a_{6}=1, a_{8}=0, k=1$, $h=-2, b_{0}=-1$, and $t=0$.

Applying the Taylor expansion results in

$$
\begin{gathered}
\phi(x, y, t)=\frac{2 a_{5}^{2} k^{2}\left(\left(1+\frac{k^{2} X^{2}}{2 !}+\ldots\right)+\left(1-\frac{k^{2} Y^{2}}{2 !}+\ldots\right)\right)}{\left(1+\frac{k^{2} X^{2}}{2 !}+\ldots\right)-\left(1-\frac{k^{2} Y^{2}}{2 !}+\ldots\right)} \\
-\frac{2\left(a_{5} k\left(\left(k X+\frac{k^{3} X^{3}}{3 !}+\ldots\right)+\left(k Y-\frac{k^{3} Y^{3}}{3 !}+\ldots\right)\right)\right)^{2}}{\left(\left(1+\frac{k^{2} X^{2}}{2 !}+\ldots\right)-\left(1-\frac{k^{2} Y^{2}}{2 !}+\ldots\right)\right)^{2}}, \\
\psi(x, y, t)=-2 a_{5} a_{6} k^{2}+\frac{2 a_{5} a_{6} k^{2}\left(\left(k X+\frac{k^{3} X^{3}}{3 !}+\ldots\right)^{2}-\left(k Y-\frac{k^{3} Y^{3}}{3 !}+\ldots\right)^{2}\right)}{\left(\left(1+\frac{k^{2} X^{2}}{2 !}+\ldots\right)-\left(1-\frac{k^{2} Y^{2}}{2 !}+\ldots\right)\right)^{2}} .
\end{gathered}
$$

Now, when $k$ tends to zero; we find a rational solution as

$$
\begin{gathered}
\phi(x, y, t)=8 a_{5}^{2}\left(\frac{1}{\mathcal{X}^{2}+\mathcal{Y}^{2}}-\frac{(\mathcal{X}+\mathcal{Y})^{2}}{\left(\mathcal{X}^{2}+\mathcal{Y}^{2}\right)^{2}}\right), \\
\psi(x, y, t)=\frac{8 a_{5} a_{6}\left(\mathcal{X}^{2}-\mathcal{Y}^{2}\right)}{\left(\mathcal{X}^{2}+\mathcal{Y}^{2}\right)^{2}}
\end{gathered}
$$

in which $\mathcal{X}=a_{5} x-a_{6} y+c_{2} a_{6} t+a_{4}$ and $\mathcal{Y}=a_{5} x+a_{6} y-c_{2} a_{6} t+a_{8}$.

The above rational solution is plotted graphically in Figure 5 for $a_{4}=0, a_{5}=-2, a_{6}=1, a_{8}=0$, and $t=0$. 

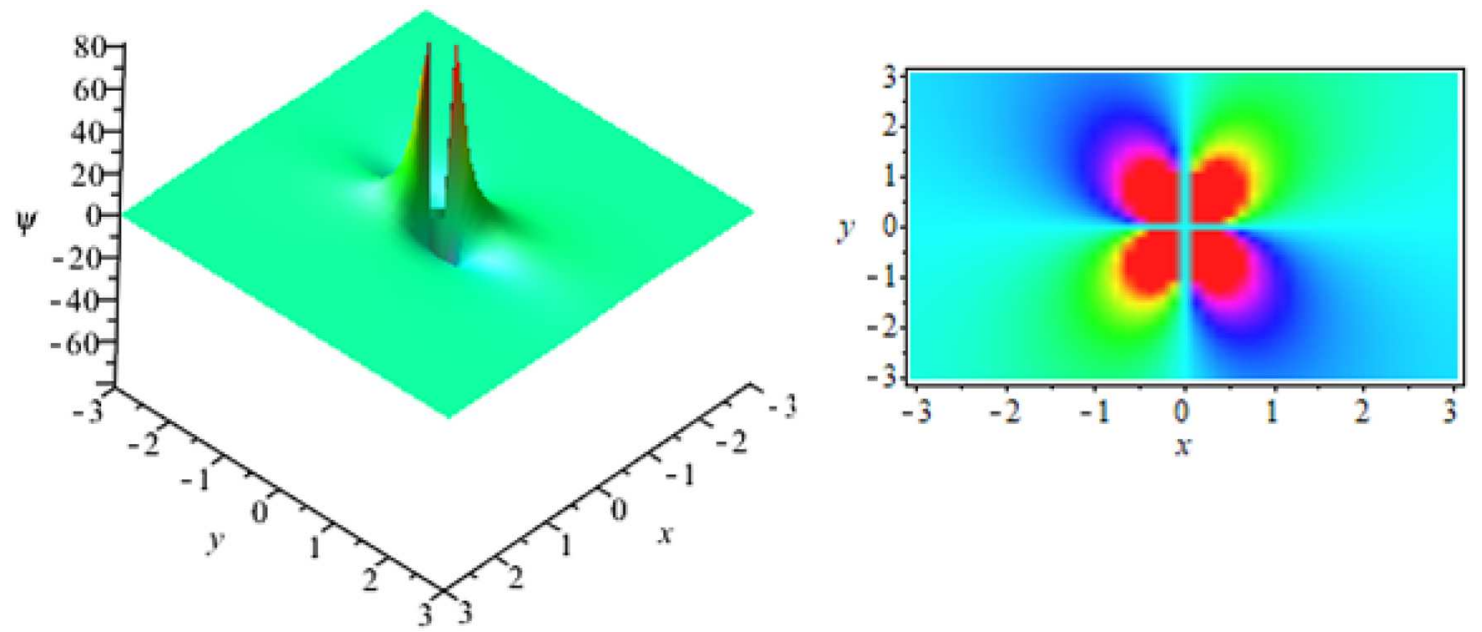

FiguRE 5. Rational solution when $a_{4}=0, a_{5}=-2, a_{6}=1, a_{8}=0$, and $t=0$.

\subsection{Complexiton solutions}

This subsection deals with complexiton solutions of the generalized 2D-HB equation. For this purpose, a test function is exerted as

$$
\varphi=1+2 e^{\theta_{1}} \cos \left(\theta_{2}\right)+a_{12} e^{2 \theta_{1}}
$$

in which

$$
\theta_{1}=k_{1} x+r_{1} y+w_{1} t, \quad \theta_{2}=k_{2} x+r_{2} y+w_{2} t
$$

and $w_{1}$ and $w_{2}$ satisfy

$P(k, r, w)=P(\bar{k}, \bar{r}, \bar{w})=0, \quad k=k_{1}+I k_{2}, \quad r=r_{1}+I r_{2}, \quad w=w_{1}+I w_{2}, \quad P=y t+c_{1} x^{3} y+c_{2} y^{2}, \quad I=\sqrt{-1}$.

Here, the polynomial $P$ has been defined owing to the Hirota operator (1.3). It is easy to prove that

$$
\begin{gathered}
w_{1}=-c_{1} k_{1}^{3}+3 c_{1} k_{1} k_{2}^{2}-c_{2} r_{1}, \\
w_{2}=c_{1} k_{2}^{3}-3 c_{1} k_{1}^{2} k_{2}-c_{2} r_{2} .
\end{gathered}
$$

The unknown $a_{12}$ also can be gained as

$$
a_{12}=-\frac{P\left(2 I k_{2}, 2 I r_{2}, 2 I w_{2}\right)}{P\left(2 k_{1}, 2 r_{1}, 2 w_{1}\right)}=-\frac{16 c_{1} k_{2}^{3} r_{2}-4 c_{2} r_{2}^{2}-4 r_{2}\left(-3 c_{1} k_{1}^{2} k_{2}+c_{1} k_{2}^{3}-c_{2} r_{2}\right)}{16 c_{1} k_{1}^{3} r_{1}+4 c_{2} r_{1}^{2}+2 r_{1}\left(-2 c_{1} k_{1}^{3}+6 c_{1} k_{1} k_{2}^{2}-2 c_{2} r_{1}\right)}
$$

Now, a complexiton solution to the generalized 2D-HB equation is obtained as

$$
\phi=2(\ln \varphi)_{x x}, \quad \psi=2(\ln \varphi)_{x y},
$$


in which

$$
\begin{gathered}
\varphi=1+2 e^{\theta_{1}} \cos \left(\theta_{2}\right)+a_{12} e^{2 \theta_{1}} \\
\theta_{1}=k_{1} x+r_{1} y+\left(-c_{1} k_{1}^{3}+3 c_{1} k_{1} k_{2}^{2}-c_{2} r_{1}\right) t, \quad \theta_{2}=k_{2} x+r_{2} y+\left(c_{1} k_{2}^{3}-3 c_{1} k_{1}^{2} k_{2}-c_{2} r_{2}\right) t \\
a_{12}=-\frac{16 c_{1} k_{2}^{3} r_{2}-4 c_{2} r_{2}^{2}-4 r_{2}\left(-3 c_{1} k_{1}^{2} k_{2}+c_{1} k_{2}^{3}-c_{2} r_{2}\right)}{16 c_{1} k_{1}^{3} r_{1}+4 c_{2} r_{1}^{2}+2 r_{1}\left(-2 c_{1} k_{1}^{3}+6 c_{1} k_{1} k_{2}^{2}-2 c_{2} r_{1}\right)} .
\end{gathered}
$$

It is worth mentioning that the following complex complexiton solution to the generalized 2D-HB equation can be extracted

$$
\phi=2(\ln \varphi)_{x x}, \quad \psi=2(\ln \varphi)_{x y}
$$

in which

$$
\begin{gathered}
\varphi=I+2 e^{\theta_{1}} \cos \left(\theta_{2}\right)-I a_{12} e^{2 \theta_{1}}, \quad I=\sqrt{-1} \\
\theta_{1}=k_{1} x+r_{1} y+\left(-c_{1} k_{1}^{3}+3 c_{1} k_{1} k_{2}^{2}-c_{2} r_{1}\right) t, \quad \theta_{2}=k_{2} x+r_{2} y+\left(c_{1} k_{2}^{3}-3 c_{1} k_{1}^{2} k_{2}-c_{2} r_{2}\right) t \\
a_{12}=-\frac{16 c_{1} k_{2}^{3} r_{2}-4 c_{2} r_{2}^{2}-4 r_{2}\left(-3 c_{1} k_{1}^{2} k_{2}+c_{1} k_{2}^{3}-c_{2} r_{2}\right)}{16 c_{1} k_{1}^{3} r_{1}+4 c_{2} r_{1}^{2}+2 r_{1}\left(-2 c_{1} k_{1}^{3}+6 c_{1} k_{1} k_{2}^{2}-2 c_{2} r_{1}\right)} .
\end{gathered}
$$

The above complexiton solution has been portrayed graphically in Figure 6 for suitable parameters.

\subsection{Hyperbolic solutions}

In this subsection, hyperbolic solutions of the generalized 2D-HB equation are obtained with the use of ansatz methods. To start, let's consider the solution of the generalized 2D-HB equation as

$$
\begin{gathered}
\phi=\mathrm{A}_{0}+\mathrm{A}_{1} \tanh (\kappa x+\tau y+\omega t)^{2}, \\
\psi=\frac{\tau}{\kappa}\left(\mathrm{A}_{0}+\mathrm{A}_{1} \tanh (\kappa x+\tau y+\omega t)^{2}\right) .
\end{gathered}
$$

By setting equations (2.53) and (2.54) in the system (1.2) and performing some calculations, we find

$$
\mathrm{A}_{1}=-2 \kappa^{2}, \quad \omega=8 c_{1} \kappa^{3}-6 \mathrm{~A}_{0} c_{1} \kappa-c_{2} \tau .
$$

Therefore, the following hyperbolic solution to the generalized 2D-HB equation is obtained

$$
\begin{gathered}
\phi=\mathrm{A}_{0}-2 \kappa^{2} \tanh \left(\kappa x+\tau y+\left(8 c_{1} \kappa^{3}-6 \mathrm{~A}_{0} c_{1} \kappa-c_{2} \tau\right) t\right)^{2}, \\
\psi=\frac{\tau}{\kappa}\left(\mathrm{A}_{0}-2 \kappa^{2} \tanh \left(\kappa x+\tau y+\left(8 c_{1} \kappa^{3}-6 \mathrm{~A}_{0} c_{1} \kappa-c_{2} \tau\right) t\right)^{2}\right) .
\end{gathered}
$$



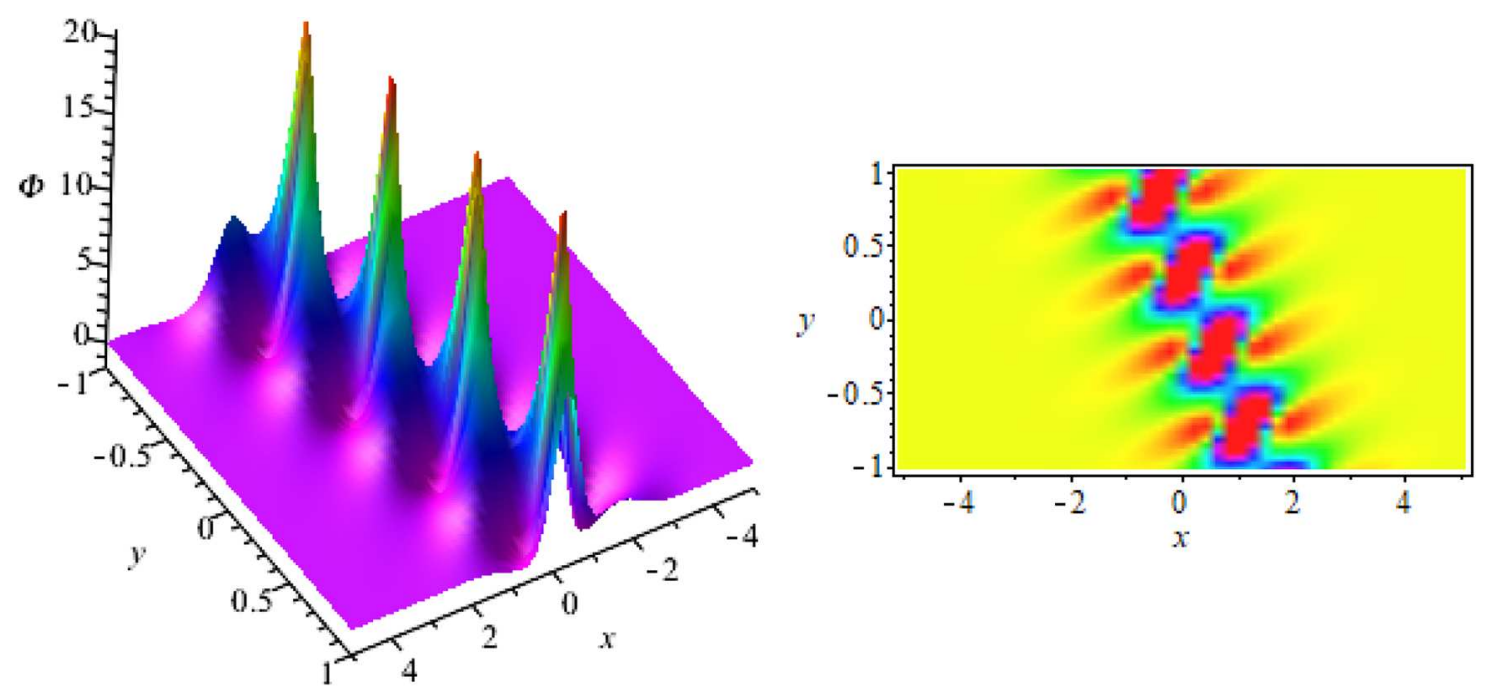

FIgURE 6. Complexiton solution when $k_{1}=-2, k_{2}=-2, r_{1}=-2, r_{2}=10$, and $t=0$.

It can be readily shown that another hyperbolic solution can be derived as

$$
\begin{gathered}
\phi=\mathrm{A}_{0}-2 \kappa^{2} \operatorname{coth}\left(\kappa x+\tau y+\left(8 c_{1} \kappa^{3}-6 \mathrm{~A}_{0} c_{1} \kappa-c_{2} \tau\right) t\right)^{2}, \\
\psi=\frac{\tau}{\kappa}\left(\mathrm{A}_{0}-2 \kappa^{2} \operatorname{coth}\left(\kappa x+\tau y+\left(8 c_{1} \kappa^{3}-6 \mathrm{~A}_{0} c_{1} \kappa-c_{2} \tau\right) t\right)^{2}\right) .
\end{gathered}
$$

Now, let us consider the following hyperbolic solution to the generalized 2D-HB equation

$$
\begin{gathered}
\phi=\mathrm{A}_{0}+\mathrm{A}_{1} \operatorname{sech}(\kappa x+\tau y+\omega t)^{2}, \\
\psi=\frac{\tau}{\kappa}\left(\mathrm{A}_{0}+\mathrm{A}_{1} \operatorname{sech}(\kappa x+\tau y+\omega t)^{2}\right) .
\end{gathered}
$$

Substituting equations (2.60) and (2.61) into the system (1.2) and performing some calculations, results in

$$
\mathrm{A}_{1}=2 \kappa^{2}, \quad \omega=-4 c_{1} \kappa^{3}-6 \mathrm{~A}_{0} c_{1} \kappa-c_{2} \tau .
$$

Therefore, the following hyperbolic solution to the generalized 2D-HB equation is derived

$$
\begin{gathered}
\phi=\mathrm{A}_{0}+2 \kappa^{2} \operatorname{sech}\left(\kappa x+\tau y+\left(-4 c_{1} \kappa^{3}-6 \mathrm{~A}_{0} c_{1} \kappa-c_{2} \tau\right) t\right)^{2}, \\
\psi=\frac{\tau}{\kappa}\left(\mathrm{A}_{0}+2 \kappa^{2} \operatorname{sech}\left(\kappa x+\tau y+\left(-4 c_{1} \kappa^{3}-6 \mathrm{~A}_{0} c_{1} \kappa-c_{2} \tau\right) t\right)^{2}\right) .
\end{gathered}
$$

In a parallel manner, it can be demonstrated that

$$
\phi=\mathrm{A}_{0}-2 \kappa^{2} \operatorname{csch}\left(\kappa x+\tau y+\left(-4 c_{1} \kappa^{3}-6 \mathrm{~A}_{0} c_{1} \kappa-c_{2} \tau\right) t\right)^{2},
$$



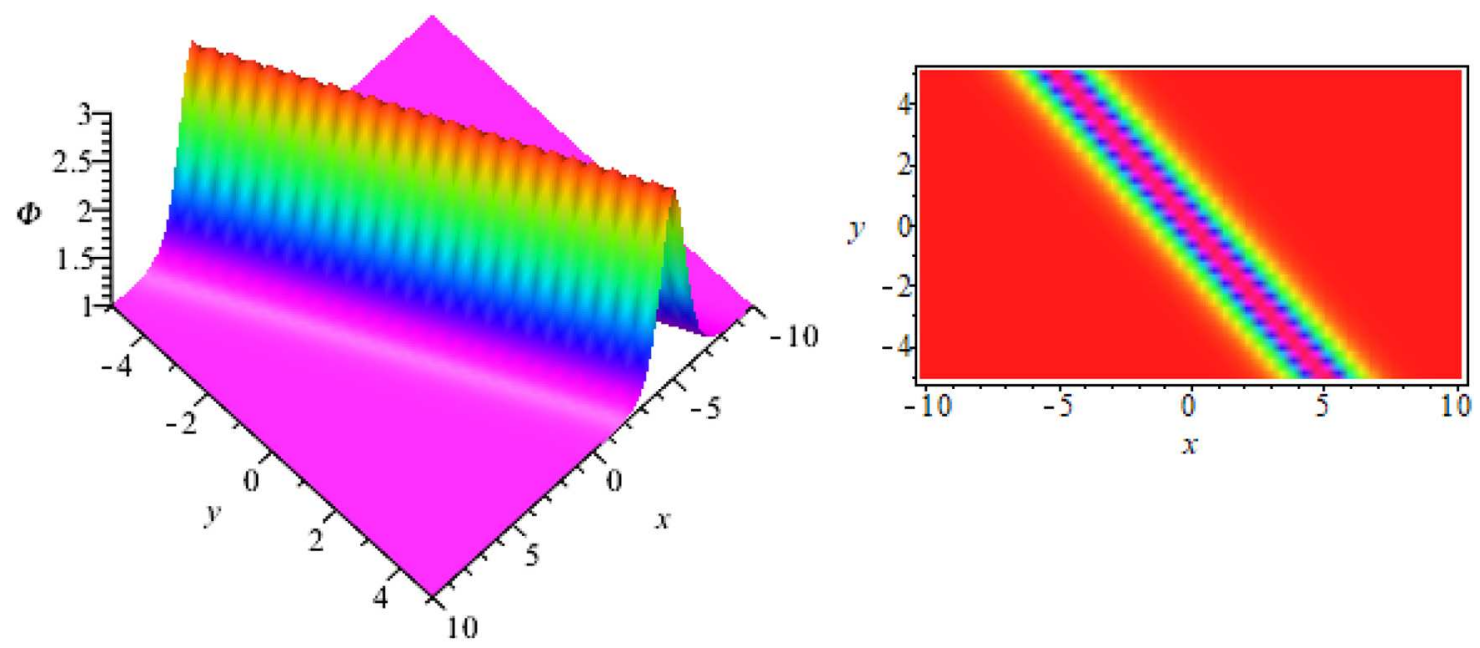

FiguRE 7. Hyperbolic solution (2.63) when $\mathrm{A}_{0}=1, \kappa=1, \tau=1$, and $t=0$.

$$
\psi=\frac{\tau}{\kappa}\left(\mathrm{A}_{0}-2 \kappa^{2} \operatorname{csch}\left(\kappa x+\tau y+\left(-4 c_{1} \kappa^{3}-6 \mathrm{~A}_{0} c_{1} \kappa-c_{2} \tau\right) t\right)^{2}\right)
$$

is the solution of the generalized 2D-HB equation.

The plots of the hyperbolic solution (2.63) have been provided in Figure 7. Obviously, Figure 7 shows a bright soliton wave solution.

Remark 2.2. Different classes of rational wave solutions have been reported in the present paper which definitely are different from the exact solutions of reference [14].

\section{CONCLUDing REMARKS}

A generalized (2+1)-dimensional Hirota bilinear equation which describes nonlinear waves in fluids and oceans was comprehensively explored, in this paper. The current goal was performed by exerting the simplified Hirota's method and ansatz approaches as reliable techniques. Different classes of rational wave structures such as multiple soliton solutions, breather solutions, rational solutions, and complexiton solutions to the generalized 2D-HB equation were successfully obtained. Physical behaviors of a series of rational wave structures were demonstrated graphically. The results of the current paper certainly helped to reveal the existence of rational wave structures of different types for the 2D-HB equation.

\section{REFERENCES}

[1] A.R. Adem, M. Mirzazadeh, Q. Zhou and K. Hosseini, Multiple soliton solutions of the Sawada-Kotera equation with a nonvanishing boundary condition and the perturbed Korteweg de Vries equation by using the multiple exp-function scheme. Adv. Math. Phys. 2019 (2019) 3175213.

[2] X.X. Du, B. Tian and Y. Yin, Lump, mixed lump-kink, breather and rogue waves for a B-type Kadomtsev-Petviashvili equation. Waves Random Complex Media (2019), doi. 10.1080/17455030.2019.1566681.

[3] L.L. Feng and T.T. Zhang, Breather wave, rogue wave and solitary wave solutions of a coupled nonlinear Schrödinger equation. Appl. Math. Lett. 78 (2018) 133-140.

[4] C.S. Gardner, J.M. Greene, M.D. Kruskal and R.M. Miura, Method for solving the Korteweg-deVries equation. Phys. Rev. Lett. 19 (1967) 1095-1097.

[5] J. He and Y. Li, Designable integrability of the variable coefficient nonlinear Schrödinger equations. Studies Appl. Math. 126 (2011) $1-15$.

[6] J. He, L. Wang, L. Li, K. Porsezian and R. Erdlyi, Few-cycle optical rogue waves: Complex modified Korteweg-de Vries equation. Phys. Rev. E 89 (2014) 062917. 
[7] J.S. He, E.G. Charalampidis, P.G. Kevrekidis and D.J. Frantzeskakis, Rogue waves in nonlinear Schrödinger models with variable coefficients: Application to Bose-Einstein condensates. Phys. Lett. A 378 (2014) 577-583.

[8] J. He, S. Xu and Y. Cheng, The rational solutions of the mixed nonlinear Schrödinger equation. AIP Adv. 5 (2015) 017105.

[9] W. Hereman and A. Nuseir, Symbolic methods to construct exact solutions of nonlinear partial differential equations. Math. Comput. Simul. 43 (1997) 13-27.

[10] W. Hereman and W. Zhuang, Symbolic software for soliton theory. Acta Appl. Math. 39 (1995) 361-378.

[11] R. Hirota, The direct method in soliton theory, Cambridge University Press, Cambridge (2004).

[12] K. Hosseini, M. Aligoli, M. Mirzazadeh, M. Eslami and J.F. Gómez-Aguilar, Dynamics of rational solutions in a new generalized Kadomtsev-Petviashvili equation. Mod. Phys. Lett. B 33 (2019) 1950437.

[13] C.C. Hu, B. Tian, H.M. Yin, C.R. Zhang and Z. Zhang, Dark breather waves, dark lump waves and lump wave-soliton interactions for a (3+1)-dimensional generalized Kadomtsev-Petviashvili equation in a fluid. Comput. Math. Appl. 78 (2019) $166-177$.

[14] Y.F. Hua, B.L. Guo, W.X. Ma and X. Lü, Interaction behavior associated with a generalized (2+1)-dimensional Hirota bilinear equation for nonlinear waves. Appl. Math. Model. 74 (2019) 184-198.

[15] M. Inc, K. Hosseini, M. Samavat, M. Mirzazadeh, M. Eslami, M. Moradi and D. Baleanu, $N$-wave and other solutions to the B-type Kadomtsev-Petviashvili equation. Ther. Sci. 23 (2019) 2027-2035.

[16] J.G. Liu, and Y. He, Abundant lump and lump-kink solutions for the new $(3+1)$-dimensional generalized KadomtsevPetviashvili equation. Nonlinear Dyn. 92 (2018) 1103-1108.

[17] J.G. Liu and Q. Ye, Stripe solitons and lump solutions for a generalized Kadomtsev-Petviashvili equation with variable coefficients in fluid mechanics. Nonlinear Dyn. 96 (2019) 23-29.

[18] J.G. Liu, J.Q. Du, Z.F. Zeng and B. Nie, New three-wave solutions for the (3+1)-dimensional Boiti-Leon-Manna-Pempinelli equation. Nonlinear Dyn. 88 (2017) 655-661.

[19] J.G. Liu, M. Eslami, H. Rezazadeh and M. Mirzazadeh, Rational solutions and lump solutions to a non-isospectral and generalized variable-coefficient Kadomtsev-Petviashvili equation. Nonlinear Dyn. 95 (2019) 1027-1033.

[20] X. Lü and W.X. Ma, Study of lump dynamics based on a dimensionally reduced Hirota bilinear equation. Nonlinear Dyn. 85 (2016) 1217-1222.

[21] W.X. Ma and Z. Zhu, Solving the (3+1)-dimensional generalized KP and BKP equations by the multiple exp-function algorithm. Appl. Math. Comput. 218 (2012) 11871-11879.

[22] W.X. Ma, T. Huang and Y. Zhang, A multiple exp-function method for nonlinear differential equations and its application. Phys. Scr. 82 (2010) 065003.

[23] M.S. Osman and A.M. Wazwaz, A general bilinear form to generate different wave structures of solitons for a (3+1)-dimensional Boiti-Leon-Manna-Pempinelli equation. Math. Methods Appl. Sci. 42 (2019) 6277-6283.

[24] M.S. Osman, M. Inc, J.G. Liu, K. Hosseini and A. Yusuf, Different wave structures and stability analysis for the generalized (2+1)-dimensional Camassa-Holm-Kadomtsev-Petviashvili equation. Phys. Scr. 95 (2020) 035229.

[25] R. Pouyanmehr, K. Hosseini, R. Ansari and S.H. Alavi, Different wave structures to the $(2+1)$-dimensional generalized Bogoyavlensky-Konopelchenko equation. Int. J. Appl. Comput. Math. 5 (2019) 149.

[26] W. Tan, H. Dai, Z. Dai and W. Zhong, Emergence and space-time structure of lump solution to the (2+1)-dimensional generalized KP equation. Pramana J. Phys. 89 (2017) 77.

[27] A.M. Wazwaz, Multiple-soliton solutions for the generalized (1+1)-dimensional and the generalized (2+1)-dimensional Ito equations. Appl. Math. Comput. 202 (2008) 840-849.

[28] A.M. Wazwaz, Multiple-soliton solutions for extended shallow water wave equations. Studies Math. Sci. 1 (2010) 21-29.

[29] A.M. Wazwaz, Two wave mode higher-order modified KdV equations: Essential conditions for multiple soliton solutions to exist. Int. J. Num. Methods Heat Fluid Flow 27 (2017) 2223-2230.

[30] A.M. Wazwaz, Multiple complex and multiple real soliton solutions for the integrable sine-Gordon equation. Optik 172 (2018) $622-627$.

[31] A.M. Wazwaz, Multiple complex soliton solutions for the integrable KdV, fifth-order Lax, modified KdV, Burgers, and SharmaTasso-Olver equations. Chin. J. Phys. 59 (2019) 372-378.

[32] A.M. Wazwaz, Multiple complex soliton solutions for integrable negative-order KdV and integrable negative-order modified KdV equations. Appl. Math. Lett. 88 (2019) 1-7.

[33] A.M. Wazwaz and L. Kaur, New integrable Boussinesq equations of distinct dimensions with diverse variety of soliton solutions. Nonlinear Dyn. 97 (2019) 83-94.

[34] R. Zhang, S. Bilige, T. Fang and T. Chaolu, New periodic wave, cross-kink wave and the interaction phenomenon for the Jimbo-Miwa-like equation. Comput. Math. Appl. 78 (2019) 754-764.

[35] Y. Zhou and W.X. Ma, Complexiton solutions to soliton equations by the Hirota method. J. Math. Phys. 58 (2017) 101511. 